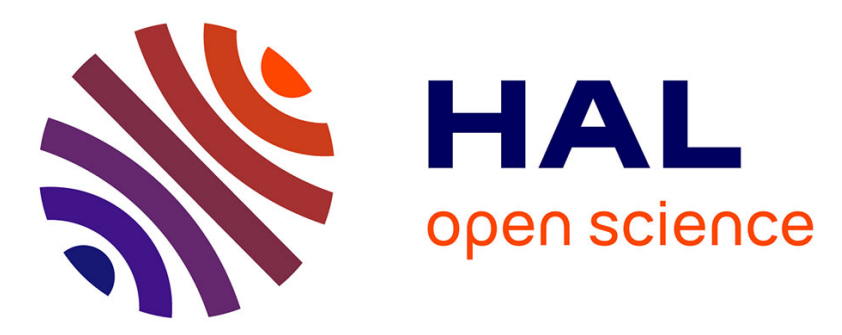

\title{
Immersion and Invariance vs Sliding Mode Control for Reference Trajectory Tracking of Autonomous Vehicles
}

\author{
Gilles Tagne, Reine Talj, Ali Charara
}

\section{To cite this version:}

Gilles Tagne, Reine Talj, Ali Charara. Immersion and Invariance vs Sliding Mode Control for Reference Trajectory Tracking of Autonomous Vehicles. European Control Conference (ECC 2014), Jun 2014, Strasbourg, France. pp.2888-2893. hal-01061134

\section{HAL Id: hal-01061134 https://hal.science/hal-01061134}

Submitted on 5 Sep 2014

HAL is a multi-disciplinary open access archive for the deposit and dissemination of scientific research documents, whether they are published or not. The documents may come from teaching and research institutions in France or abroad, or from public or private research centers.
L'archive ouverte pluridisciplinaire HAL, est destinée au dépôt et à la diffusion de documents scientifiques de niveau recherche, publiés ou non, émanant des établissements d'enseignement et de recherche français ou étrangers, des laboratoires publics ou privés. 


\title{
Immersion and Invariance vs Sliding Mode Control for Reference Trajectory Tracking of Autonomous Vehicles
}

\author{
Gilles Tagne, Reine Talj and Ali Charara
}

\begin{abstract}
This paper focuses on the lateral control of intelligent vehicles. The aim is to minimize the lateral displacement of the autonomous vehicle with respect to a given reference trajectory. We present design and validation of two vehicle lateral controllers. The first controller based on higher-order sliding mode control (SMC) has been developed in [1]. The second controller is based on Immersion and Invariance $(I \& I)$ principle. Given the implicit resemblance between the SMC and the $I \& I$ principle, the comparison allows us to highlight the advantages and disadvantages of each control strategy for robust lane keeping for intelligent vehicles. To validate the control strategies, the closed-loop system has been simulated on Matlab-Simulink has been made using the experimental data acquired on the vehicle DYNA of Heudiasyc laboratory, a Peugeot 308, according to several real driving scenarios. The validation shows robustness and performances of each control approach, and puts in evidence the improvement brought by the $I \& I$ controller.
\end{abstract}

\section{INTRODUCTION}

The development of Advanced Driver Assistance Systems (ADAS) and autonomous driving has been favored by technological advances in recent years. Three main steps are necessary to ensure an autonomous navigation: the perceptionlocalization, the path planning and the vehicle control. The vehicle control can be divided into two tasks: longitudinal control and lateral control. This paper focuses on the lateral control of intelligent vehicles. This is a very active research field that has been studied since the 1950s.

Lateral control consists on handling the vehicle using the steering wheel to follow the reference trajectory. Given the high nonlinearity of the vehicle on one hand, and the uncertainties and disturbances in automotive applications on the other hand, a very important issue to be considered in the control design is the robustness. The controller should be able to reject disturbances and deal with parameter uncertainties and variations. For example, in [2], a recent presentation of Junior; Stanford's autonomous research vehicle (the second at the DARPA Urban Challenge) is made for the purpose of ensuring robust autonomous driving.

In recent years, considerable research has been made to provide lateral guidance of autonomous vehicles. In literature, many control strategies have been developed. Simple

This work was carried out in the framework of the Labex MS2T, which was funded by the French Government, through the program Investments for the future managed by the National Agency for Research (Reference ANR-11-IDEX-0004-02)

The authors are working at Heudiasyc Laboratory, UMR CNRS 7253, Université de Technologie de Compiègne, BP 20529, 60205 Compiègne, France

gilles.tagne@hds.utc.fr, reine.talj@hds.utc.fr, ali.chararaehds.utc.fr
PID controllers have been proposed in [3] and [4]. We also have nested one in [5]. Moreover, other classical techniques have been used. We can cite $H_{\infty}$ [6], state feedback [7], Lyapunov stability based control [8], fuzzy logic [9], fuzzy Takagi-Sugeno LQ [10], linear quadratic optimal predictive control [11], backstepping based approach [12] and many others. On the other hand, Model Predictive Control (MPC) appears to be well suited to the trajectory following [2], [13]. In [14] and [1], Sliding Mode Control (SMC) has been applied to the lateral control. This robust control strategy is well suited to driving applications, given its robustness against uncertainties and its capacity to reject disturbances. However, its main drawback is the chattering.

A variety of different vehicle controllers was proposed for trajectory tracking. Most of the works published previously do not compare their results with other approaches. Unfortunately, comparisons between existing control concepts are very rare. In [15], a comparison was made between proportional, adaptive, $H_{\infty}$ and fuzzy controllers. More recently, in [16], a comparison of two controllers for trajectory tracking emergency was performed. In [17], continuous-time and discrete-time switched $H_{\infty}$ were compared. It is therefore difficult to make an objective classification from the literature but different results showed clearly that the class of adaptive controllers represents a very promising technique for such uncertain and nonlinear application.

In [1], we have developed a controller based on higherorder sliding mode control. Given the implicit resemblance between the sliding mode controller and the I\&I principle, for the purpose of designing nonlinear and adaptive controllers, a controller based on the $I \& I$ approach is developed for a comparative study.

The $I \& I$ principle is a relatively new method for designing nonlinear and adaptive controllers. The method relies upon the notions of system immersion and manifold invariance. The basic idea of the $I \& I$ approach is to achieve the control objective by immersing the plant dynamics into a (possibly lower-order) target system that captures the desired behavior [18]. This is achieved by finding a manifold in state-space that can be rendered invariant and attractive; with internal dynamics that reflect the desired closed-loop dynamics and by designing a control law that takes the state of the system towards the manifold. In others words, the $I \& I$ theory consists of defining a target dynamics and to design a control law that renders the manifold of the target dynamics attractive and invariant. Indeed, the $I \& I$ reformulation of the stabilization problem is implicit in sliding mode control, where the target dynamics are the 
dynamics of the system on the sliding surface, which is made attractive by a discontinuous control law.

In this paper, the design of both controllers is presented. Moreover, the $I \& I$ method allows to prove a very strong stability criterion of the closed-loop system. In fact, for all controller gains chosen to be positive, the closed-loop system is stable. To design the controller, we consider that the vehicle is equipped with sensors or observers to measure sideslip angle, yaw rate, lateral error and its derivative. Both controllers use the same signals (variables). To validate the control laws, tests are made with real data acquired on the vehicle DYNA, on the tracks and circuits of CERAM ${ }^{1}$. The simulation results show the performance and robustness of the proposed approaches and put in evidence the improvement brought by the $I \& I$ controller.

This paper is organized as follows. Section II presents the dynamical models of the vehicle, used for control design and validation. In Section III, the control problem definition is presented. The design of the SMC and the $I \& I$ controllers is made in Section IV. Section V presents the simulation results. Finally, we conclude in Section VI, with some remarks and future work directions.

\section{Dynamic Models of Vehicle}

In this work, we use two vehicle models. To design the controller, a simple and widely used dynamic bicycle model [7] is considered (see Fig. 1).

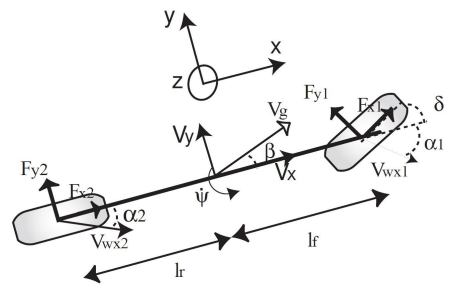

Fig. 1. Bicycle model

This model is used to represent the lateral vehicle behavior and assumes that the vehicle is symmetrical, and tire sideslip angles on the same axle are equal. The roll and pitch dynamics are neglected and angles are assumed to be small (steering, sideslip, yaw). With a linear tire force model we obtain a Linear Parameter Varying (LPV) model, where the longitudinal velocity $V_{x}$ is considered as a varying parameter. Dynamic equations in terms of sideslip angle and yaw rate of the bicycle model are given by:

$$
\left\{\begin{array}{l}
\dot{\beta}=-\frac{\left(C_{f}+C_{r}\right)}{m V_{x}} \beta-\left(1+\frac{L_{f} C_{f}-L_{r} C_{r}}{m V_{x}^{2}}\right) \dot{\psi}+\frac{C_{f}}{m V_{x}} \delta \\
\ddot{\psi}=-\frac{L_{f} C_{f}-L_{r} C_{r}}{I_{z}} \beta-\frac{L_{f}^{2} C_{f}+L_{r}^{2} C_{r}}{I_{z} V_{x}} \dot{\psi}+\frac{L_{f} C_{f}}{I_{z}} \delta
\end{array}\right.
$$

where $\beta, \psi$ and $\delta$ represent respectively the sideslip angle, the yaw angle of the vehicle and the steering wheel angle (control input). Table I presents the vehicle parameters and nomenclature.

To validate in simulation the proposed controllers in closed-loop, we used a more representative model, namely

\footnotetext{
${ }^{1}$ CERAM -"Centre d'Essais et de Recherche Automobile de Mortefontaine" is an automobile testing and research center located in France.
}

TABLE I

Vehicle Parameters and Nomenclature (Bicycle Model)

\begin{tabular}{|c||c||c||c|}
\hline$V_{x}$ & Longitudinal velocity & - & {$[\mathrm{m} / \mathrm{s}]$} \\
\hline$\beta$ & Sideslip angle & - & {$[\mathrm{rad}]$} \\
\hline$\dot{\psi}$ & Yaw rate & - & {$[\mathrm{rad} / \mathrm{s}]$} \\
\hline$\delta$ & Steering wheel angle & - & {$[\mathrm{rad}]$} \\
\hline $\mathrm{m}$ & Mass & 1719 & {$[\mathrm{~kg}]$} \\
\hline$I_{z}$ & Yaw moment of inertia & 3300 & {$\left[\mathrm{kgm}^{2}\right]$} \\
\hline$L_{f}$ & Front axle-COG distance & 1.195 & {$[\mathrm{~m}]$} \\
\hline$L_{r}$ & Rear axle-COG distance & 1.513 & {$[\mathrm{~m}]$} \\
\hline$C_{f}$ & Cornering stiffness of the front tire & 170550 & {$[\mathrm{~N} / \mathrm{rad}]$} \\
\hline$C_{r}$ & Cornering stiffness of the rear tire & 137844 & {$[\mathrm{~N} / \mathrm{rad}]$} \\
\hline
\end{tabular}

the 4-wheel model to represent the behavior of the vehicle and Dugoff's tire model for longitudinal and lateral tire forces.

\section{CONTROL PROBLEM DEFINITION}

The aim of the lateral control of autonomous intelligent vehicles is to minimize the lateral displacement of the vehicle with respect to a given reference path. The lateral error dynamics at the center of gravity of the vehicle, with respect to a reference path, is given by $\ddot{e}=a_{y}-a_{y_{r e f}}$; where $a_{y}$ and $a_{y_{\text {ref }}}$ represent respectively the lateral acceleration of the vehicle, and the desired one on the reference path. Assuming that the desired lateral acceleration can be written as $a_{y_{\text {ref }}}=V_{x}^{2} \rho$, where $\rho$ is the curvature of the road and given that $a_{y}=V_{x}(\dot{\beta}+\dot{\psi})$ [7], one can find:

$$
\ddot{e}=V_{x}(\dot{\beta}+\dot{\psi})-V_{x}^{2} \rho
$$

Replacing $\dot{\beta}$ by its expression in equation (1), we obtain:

$$
\ddot{e}=-\frac{C_{f}+C_{r}}{m} \beta-\frac{L_{f} C_{f}-L_{r} C_{r}}{m V_{x}} \dot{\psi}-V_{x}^{2} \rho+\frac{C_{f}}{m} \delta
$$

The new system state variables are $x=(\beta, \dot{\psi}, \dot{e}, e)^{\top}$, corresponding to the sideslip angle, the yaw rate, the lateral error and its derivative. The system has the following dynamics:

$$
\dot{x}=A x+B_{1} \delta+B_{2} \rho
$$

where,

$$
\begin{gathered}
A=\left[\begin{array}{cccc}
-\frac{\left(C_{f}+C_{r}\right)}{m V_{x}} & -1-\frac{\left(L_{f} C_{f}-L_{r} C_{r}\right)}{m V_{x}^{2}} & 0 & 0 \\
-\frac{\left(L_{f} C_{f}-L_{r} C_{r}\right)}{I_{z} C_{r}} & -\frac{\left(L_{f}^{2} C_{f}+L_{r}^{2} C_{r}\right)}{I_{z} V_{x}} & 0 & 0 \\
-\frac{\left(C_{f}+C_{r}\right)}{m} & -\frac{\left(L_{f} C_{f}-L_{r} C_{r}\right)}{m V_{x}} & 0 & 0 \\
0 & 0 & 1 & 0
\end{array}\right] \\
B_{1}=\left[\begin{array}{c}
\frac{C_{f}}{m V_{x}} \\
\frac{L_{f} C_{f}}{I_{z}} \\
\frac{C_{f}}{m} \\
0
\end{array}\right], \quad B_{2}=\left[\begin{array}{c}
0 \\
0 \\
-V_{x}^{2} \\
0
\end{array}\right]
\end{gathered}
$$

The aim of the lateral control is to cancel the lateral displacement error. Then, for a given curvature $\rho$ and longitudinal velocity $V_{x}$, the desired behavior corresponds to $\dot{e}=e=0$. Hence, it is easy to prove that the desired equilibrium point is [7]:

$$
(\beta, \dot{\psi}, \dot{e}, e)^{\top}=\left(\beta^{\star}, \dot{\psi}^{\star}, 0,0\right)^{\top}
$$


with

$$
\begin{aligned}
& \beta^{\star}=\left(L_{r}-\frac{L_{f} m V_{x}^{2}}{C_{r}\left(L_{f}+L_{r}\right)}\right) \rho \\
& \dot{\psi}^{\star}=V_{x} \rho
\end{aligned}
$$

At the equilibrium point, the control input is:

$$
\delta^{\star}=\left(L_{f}+L_{r}\right) \rho+\frac{m V_{x}^{2}\left(L_{r} C_{r}-L_{f} C_{f}\right)}{C_{f} C_{r}\left(L_{f}+L_{r}\right)} \rho
$$

We define the new error variables:

$$
\left\{\begin{array}{l}
\tilde{\beta}=\beta-\beta^{\star} \\
\dot{\tilde{\psi}}=\dot{\psi}-\dot{\psi}^{\star} \\
\tilde{\delta}=\delta-\delta^{\star}
\end{array}\right.
$$

Hence, the error dynamics of the system (4) having the origin as equilibrium point $(\tilde{\beta}, \dot{\tilde{\psi}}, \dot{e}, e)^{\top}=(0,0,0,0)^{\top}$ become:

$$
\dot{\tilde{x}}=A \tilde{x}+B_{1} \tilde{\delta}
$$

where, $A$ and $B_{1}$ have been defined above (5).

\section{SMC AND I\&I CONTROLLERS DESIGN}

After a brief presentation of the SMC controller, we will present the design of the $I \& I$ controller.

\section{A. SMC controller}

The SMC has been developed since 1950s and is recognized as one of the most promising techniques for robust control. The principle of SMC is to constrain the system trajectories to reach in finite time and remain on a sliding surface. However, its main drawback is the chattering. In this subsection, we recall the main lines of the design of the SMC controller. We used the super-twisting algorithm to minimize the lateral displacement. For more details, see [1].

The objective is to cancel the lateral displacement error. To this end, choosing the sliding variable $s$ as follows:

$$
s=\dot{e}+\lambda e
$$

we obtain: $\dot{s}=\ddot{e}+\lambda \dot{e}$. Replacing $\ddot{e}$ by its expression (3), we obtain:

$$
\dot{s}=-\frac{C_{f}+C_{r}}{m} \beta-\frac{L_{f} C_{f}-L_{r} C_{r}}{m V_{x}} \dot{\psi}-V_{x}^{2} \rho+\frac{C_{f}}{m} \delta+\lambda \dot{e}
$$

The variable $s$ has a relative degree $r=1$ with respect to the input $\delta$. We can write $\dot{s}$, of the form:

$$
\dot{s}(t, s)=\phi(t, s)+\varphi(t, s) \delta
$$

with,

$$
\left\{\begin{array}{l}
\phi(t, s)=-\frac{C_{f}+C_{r}}{m} \beta-\frac{L_{f} C_{f}-L_{r} C_{r}}{m V_{x}} \dot{\psi}-V_{x}^{2} \rho+\lambda \dot{e} \\
\varphi(t, s)=\frac{C_{f}}{m}
\end{array}\right.
$$

It is assumed that there exist positive constants $s_{0}, b_{\min }, b_{\max }$, $C_{0}$ such that $\forall x \in \mathbb{R}^{n}$ and $|s(t, x)|<s_{0}$, the system satisfies the following conditions:

$$
\left\{\begin{array}{l}
|\delta| \leq \delta_{\max } \\
0<b_{\min } \leq|\varphi(t, s)| \leq b_{\max } \\
|\phi(t, s)|<C_{0}
\end{array}\right.
$$

So, the SMC algorithm based on super-twinting is given by:

$$
\delta_{S T}=\delta_{1}+\delta_{2}\left\{\begin{array}{l}
\delta_{1}=-\alpha_{1}|s|^{1 / 2} \operatorname{sign}(s) \\
\dot{\delta}_{2}=-\alpha_{2} \operatorname{sign}(s)
\end{array}\right.
$$

with $\alpha_{1}$ and $\alpha_{2}$ positive constants. The finite time convergence to the sliding surface is guaranteed for all:

$$
\left\{\begin{array}{l}
\alpha_{2}>\frac{C_{0}}{b_{\min }} \\
\alpha_{1} \geq \sqrt{\frac{4 C_{0}\left(b_{\max } \alpha_{2}+C_{0}\right)}{b_{\min }^{2}\left(b_{\min } \alpha_{2}-C_{0}\right)}}
\end{array}\right.
$$

To avoid important peaks in transient phases, we add an equivalent command $\delta_{e q}$ obtained by solving the equation $\dot{s}=0$. This term has the role of a feedforward that brings the system near to the sliding surface, and is given by:

$$
\delta_{e q}=-\frac{m}{C_{f}} \phi(t, s)
$$

Hence, the steering angle representing the control input of the system is defined as follows:

$$
\delta_{S M C}=\delta_{S T}+\delta_{e q}
$$

\section{B. I\&I controller}

The principle of the $I \& I$ consists to achieving the control objective by immerging the system into a target dynamics that ensures the objective of the command. To design the $I \& I$ controller, some assumptions should be verified: the system and the target dynamics should both have their equilibrium at the origin respectively. The immersion of the system is achieved by an attractive and invariant manifold. So, the command consists on bringing the system state to the manifold. Astolfi et al. [18] presents the main stabilization result of the Immersion and Invariance method.

Now consider the vehicle lateral dynamical model (9). As mentioned before, the main objective of the steering controller is to cancel the lateral error displacement with respect to a given trajectory, i.e. $e=\dot{e}=0$ at the equilibrium.

The target system is consequently chosen to be the image of the system (9) when $e=\dot{e}=0$. More precisely, the target state vector is $\left(\xi_{1}, \xi_{2}\right)$, where $\xi_{1}$ and $\xi_{2}$ represent $\tilde{\beta}$ and $\dot{\tilde{\psi}}$ respectively, when $e=\dot{e}=0$. Note that for $e=\dot{e}=0$, we also have $\ddot{e}=0$. The dynamics can be expressed as follows,

$$
\left[\begin{array}{l}
\dot{\xi}_{1} \\
\dot{\xi}_{2}
\end{array}\right]=\left[\begin{array}{cc}
0 & -1 \\
\frac{C_{r}\left(L_{f}+L_{r}\right)}{I_{z}} & -\frac{L_{r} C_{r}\left(L_{f}+L_{r}\right)}{I_{z} V_{x}}
\end{array}\right]\left[\begin{array}{l}
\xi_{1} \\
\xi_{2}
\end{array}\right]
$$

Proposition 1: The target model (19) has a globally asymptotically stable equilibrium at the origin $(0,0)$.

Proof: The dynamics of the state vector $\xi=\left[\xi_{1}, \xi_{2}\right]$ can be written in the form $\dot{\xi}=C \xi$, with

$$
C=\left[\begin{array}{cc}
0 & -1 \\
\frac{C_{r}\left(L_{f}+L_{r}\right)}{I_{z}} & -\frac{L_{r} C_{r}\left(L_{f}+L_{r}\right)}{I_{z} V_{x}}
\end{array}\right]
$$

Then, after some simple calculations, we obtain:

$$
\operatorname{det}(s I-C)=s^{2}+\frac{L_{r} C_{r}\left(L_{f}+L_{r}\right)}{I_{z} V_{x}} s+\frac{C_{r}\left(L_{f}+L_{r}\right)}{I_{z}}
$$

The matrix $C$ verifies the Routh-Hurwitz stability criterion $\left(V_{x}>0\right)$, which yields to the desired result. 
Proposition 2: Consider the system (9) having the equilibrium point at the origin. Moreover, the subsystem (19), which is the image of the system (9) for $e=\dot{e}=0$, has a globally asymptotically stable equilibrium at the origin. Then, the system (9) is $I \& I$-stabilisable with target dynamics (19).

Proof: We define now the off-the-manifold variable

$$
z=\dot{e}+\lambda e, \quad \text { s.t. } \lambda>0
$$

Note that we choose $z=s$, the sliding variable defined above for the design of the SMC controller.

We have to select a control input $\tilde{\delta}$ such that the trajectories of the closed-loop system are bounded and $z=\dot{e}+\lambda e$ converges to zero. Notice that, when $z \rightarrow 0$, $e$ converges exponentially to zero with the rate of convergence $\lambda$, yielding to the desired result. There is a strong resemblance with the principle of SMC which is to constrain the system trajectories to reach in finite time and remain on a sliding surface $s$. In the SMC, the convergence in finite time is guaranteed by a special law (15) with respect to certain conditions (16). For the $I \& I$ controller, we choose an exponential convergence. To this end, let

$$
\dot{z}=-K z, \quad \text { with } K>0 \text {. }
$$

where $K$ represents the rate of exponential convergence of $z$ to zero. Replacing $\dot{z}$ and $z$ by their expressions in (23), and after some calculations, one can find that the corresponding control input has the following expression:

$\tilde{\delta}=-\frac{m(K+\lambda)}{C_{f}} \dot{e}-\frac{m K \lambda}{C_{f}} e+\frac{C_{f}+C_{r}}{C_{f}} \tilde{\beta}+\frac{L_{f} C_{f}-L_{r} C_{r}}{C_{f} V_{x}} \dot{\tilde{\psi}}$

The closed-loop system becomes:

$$
\left[\begin{array}{c}
\dot{\tilde{\beta}} \\
\ddot{\tilde{\psi}} \\
\ddot{e} \\
\dot{e}
\end{array}\right]=\left[\begin{array}{l|l}
\mathscr{A}_{11} & \mathscr{A}_{12} \\
\hline \mathscr{A}_{21} & \mathscr{A}_{22}
\end{array}\right]\left[\begin{array}{c}
\tilde{\beta} \\
\dot{\tilde{\psi}} \\
\dot{e} \\
e
\end{array}\right]
$$

with,

$$
\begin{aligned}
& \mathscr{A}_{11}=\left[\begin{array}{cc}
0 & -1 \\
\frac{C_{r}\left(L_{f}+L_{r}\right)}{I_{z}} & -\frac{L_{r} C_{r}\left(L_{f}+L_{r}\right)}{I_{z} V_{x}}
\end{array}\right]=C, \\
& \frac{-(K+\lambda)}{V_{x}} \\
& \mathscr{A}_{12}=\left[\begin{array}{cc}
L_{x} \\
-\frac{L_{f} m(K+\lambda)}{I_{z}} & -\frac{L_{f} m K \lambda}{I_{z}}
\end{array}\right], \\
& \mathscr{A}_{21}=\left[\begin{array}{cc}
0 & 0 \\
0 & 0
\end{array}\right], \\
& \mathscr{A}_{22}=\left[\begin{array}{cc}
-(K+\lambda) & -K \lambda \\
1 & 0
\end{array}\right] .
\end{aligned}
$$

The closed-loop system can be interpreted as the interconnection of two subsystems: $S_{1}$ with the state variables $\left(\tilde{\beta}\right.$ and $\dot{\tilde{\psi}}$ ), and $S_{2}$ (with the state variables $\dot{e}$ and $e$ ). Given that the interaction matrix $\mathscr{A}_{21}$ is identically zero, hence, the dynamics of the subsystem $S_{2}$ are independent of $S_{1}$, and can be written as follows:

$$
\left[\begin{array}{c}
\ddot{e} \\
\dot{e}
\end{array}\right]=\left[\begin{array}{cc}
-(K+\lambda) & -K \lambda \\
1 & 0
\end{array}\right]\left[\begin{array}{l}
\dot{e} \\
e
\end{array}\right]
$$

The subsystem $S_{2}$ combines and represents the interactions between the dynamics of both off-the-manifold variables $z$ and $e$, which ensure the convergence of $e$ to zero.

Proposition 3: The subsystem $S_{2}$ has a globally asymptotically stable equilibrium at the origin $(0,0)$.

Proof: The dynamics of the state vector $\zeta=[\dot{e}, e]$ can be written in the form : $\dot{\zeta}=\mathscr{A}_{22} \zeta$. The matrix $\mathscr{A}_{22}$ verifies the Routh-Hurwitz stability criterion for all $\lambda>0$ and $K>0$, hence the attractivity of the manifold is ensured. Consequently, the rates of convergence $K$ and $\lambda$ of $z$ and $e$ respectively, have to be chosen based on other practical considerations related to the system.

The subsystem $S_{1}$ depends on $S_{2}$ via the matrix $\mathscr{A}_{12}$. Defining $\tilde{u}=(K+\lambda) \dot{e}+K \lambda e$, the subsystem $S_{1}$ in closedloop has the form:

$$
\left[\begin{array}{c}
\dot{\tilde{\beta}} \\
\ddot{\tilde{\psi}}
\end{array}\right]=C\left[\begin{array}{c}
\tilde{\beta} \\
\tilde{\tilde{\psi}}
\end{array}\right]+\left[\begin{array}{c}
-\frac{1}{V_{x}} \\
-\frac{L_{f} m}{I_{z}}
\end{array}\right] \tilde{u}
$$

Finally it is clear that when $S_{2}$ converges to $(0,0)$, the input $\tilde{u}$ of the subsystem $S_{1}$ converges to zero. Then, the system $S_{1}$ converges to the target dynamics (19), which is stable and converges to $(0,0)$. Indeed, we have proved previously that this target model has a globally asymptotically stable equilibrium at the origin $(0,0)$. So, the trajectories of the closed-loop system are bounded, yielding to the desired result. For more details on the proof of global asymptotic stability of the closed loop system, see [19], which presents that the cascade of Global Asymptotic Stable systems (GAS) with a bounded trajectory gives an global asymptotic stable system. In other words, GAS + GAS + bounded trajectory $=$ GAS.

Stability of the system is assured with the proposed $I \& I$ controller for all its gains $\lambda>0$ and $K>0$. This is a very strong result that reveals some interesting characteristics of the system. Finally, the control input applied to the system (4) is:

$$
\begin{aligned}
\delta_{I \& I}=\tilde{\delta}+\delta^{\star}= & -\frac{m(K+\lambda)}{C_{f}} \dot{e}-\frac{m K \lambda}{C_{f}} e+\frac{C_{f}+C_{r}}{C_{f}} \beta \\
& +\frac{L_{f} C_{f}-L_{r} C_{r}}{C_{f} V_{x}} \dot{\psi}+\frac{m V_{x}^{2}}{C_{f}} \rho
\end{aligned}
$$

The design of this controller allows us to prove that the stability of the controller is guaranteed for all positive gains. This result helps us to show certain characteristics of passivity of the system. These structural features of the system help to improve the design of the controller. Moreover, the response time of the controller is entirely determined by the parameters $K$ and $\lambda$, which is not the case for the SMC controller. Finally, the design of this controller guarantees robust stability and some a priori known performances (response time of the controller).

\section{RESULTS}

To validate our control laws, the used experimental data were acquired on the vehicle DYNA on the CERAM test circuits. This vehicle is equipped with different sensors or observers providing the dynamic variables of the vehicle and its traveled path. The simulations in closed-loop are 
performed using the real data, as a reference with the full vehicle model. For the control laws, we used the gains $\lambda=8$ and $K=1$ for the $I \& I$ controller, and $\lambda=8, \alpha_{1}=$ $0.005, \alpha_{2}=0.002$ for the SMC controller, with the nominal vehicle parameters (see Table I).

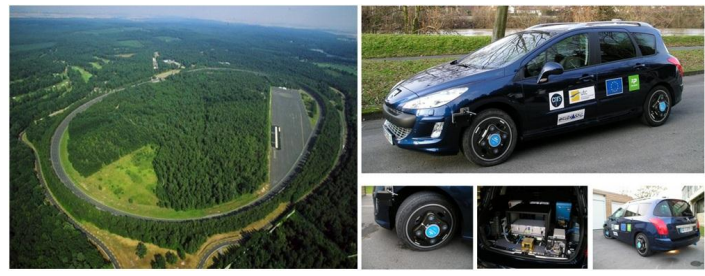

Fig. 2. Experimental vehicle (DYNA)

\section{A. Test of the controllers during normal driving with nominal parameters}

In this test (Fig. 3), lateral acceleration is less than $4 \mathrm{~m} / \mathrm{s}^{2}$. Longitudinal velocity is almost constant $(13.5 \mathrm{~m} / \mathrm{s})$. Fig. 3 shows the reference path and the trajectories followed by the controlled vehicles, the longitudinal speed variations and the lateral error. We observe that both controllers can
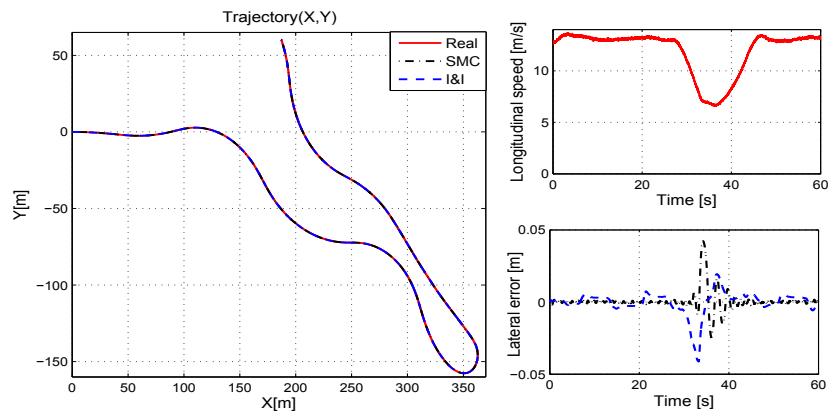

Fig. 3. Longitudinal speed, trajectories and lateral error

ensure the convergence of the lateral error. After the transient periods (change in curvature of the road), the SMC controller converges to 0 while the $I \& I$ controller converges to a small but nonzero value. The convergence time of the SMC controller is lower than that of the $I \& I$ controller. Both controllers have maximum comparable errors and ensure trajectory tracking (variable curvature).

Several other tests were performed, including scenarios of driving at high speed as well as situations where there is a saturation of lateral forces. So, tests show the good performance of both control strategies during normal driving at high and varying speed for nominal parameters known.

\section{B. Robustness of controllers to vehicle parameter uncertain- ties}

As mentioned previously, one of the major challenges for trajectory tracking is the robustness of the controller. In this subsection, we evaluate the robustness of the controllers with respect to parametric uncertainties of the vehicle. It is important to note that the parametric uncertainties can be due to the fact that the parameters may vary, but are considered to be fixed for the command, for example the mass of the vehicle.

It is difficult to estimate accurately the cornering stiffness of the tire. Moreover, this parameter varies greatly depending on the type of the road, the vertical load, the camber, etc. It is therefore important to assess the robustness of the controller over cornering stiffness variations. Fig. 4 presents lateral errors for different uncertainties on the value of the cornering stiffness for the previous test.
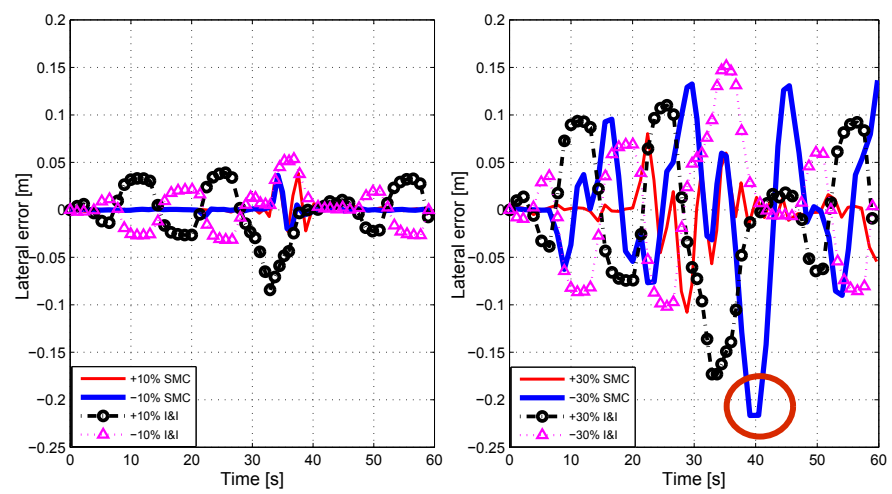

Fig. 4. Robustness against uncertainties of cornering stiffness

For uncertainties in the order of $+/-30 \%$, on the value of cornering stiffness, both controllers are able to follow the path with acceptable errors. In other words, the controllers are able to track the trajectory with a road coefficient of friction of 0.7 (cornering stiffness proportional to road coefficient of friction). Therefore, the robustness of the controller against cornering stiffness variations implicitly allows us to evaluate the robustness with respect to an unknown road coefficient of friction.

For uncertainties in the order of $10 \%$ on the value of cornering stiffness or mass, the SMC controller have similar errors (to those of nominal conditions). With the $I \& I$ controller, the error depends on the value of the parametric uncertainty. Note that the value of the parameter uncertainty strongly influences the convergence time of the SMC controller. When parametric uncertainty exceeds a certain threshold, the convergence over time is not guaranteed, and the errors of the SMC controller increases with great rates (see Fig. 4, SMC +/-30\%).

To summarize, we observe that the SMC controller provided low and almost constant errors while the condition of convergence in finite time is satisfied. When parametric uncertainty exceeds a certain threshold, the convergence in finite time is not guaranteed, and the error increases with a very large rate. It is important to note that this threshold is not known a priori and depends on the parameters, variables of the system and the amplitudes of the noises in the data. By increasing the values of the SMC controller gains to ensure acceptable errors for large parametric variations, one obtains an excessive increase in the phenomenon of chattering. This has the effect of degrading the passenger comfort. Also, it is important to note that in practice it is limited by the 
bandwidth of the steering actuator. In this validation, we use a steering actuator with a cut-off frequency of $10 \mathrm{~Hz}$. For reasons of comfort we could impose a lower frequency, which would has the effect of degrading the performance of the SMC.

With the $I \& I$ controller, the error depends on the value of the parametric uncertainty and remains acceptable even for large variations. We note that large variations in the parameters degrade the performance but stability is maintained. This clearly shows that we have a robust stability (not depending on the value of the parameters of the system). Finally, these results show robustness and performances of each control approach.

\section{CONCLUSION}

In this paper, we have compared two controllers for trajectory tracking of an autonomous vehicle. The first controller is the higher order sliding mode and specifically the super-twisting algorithm. The second controller is based on the Immersion and Invariance principle. Given the great similarity between these two control strategies, we aimed to improving the sliding mode by using the Immersion and Invariance principle (reduction or elimination of chattering, reduction of the sensitivity to the dynamic of the actuator, greater taking into account of the model).

The design of both controllers has been presented. The SMC controller has shown better performance for small parameter variations, while the condition of convergence in finite time is satisfied. This condition depends on the value of the system parameters and noise. It is therefore difficult to tune this controller. The design of the $I \& I$ controller allowed us to prove that the stability of this controller is guaranteed for all positive gains $(\lambda>0$ and $K>0)$. In addition, the response time of that controller is totally determined by the parameters $K$ and $\lambda$. So, performance and tuning of this controller is easily determined. This controller is able to give acceptable errors for large parametric uncertainties and guarantees a comfortable ride (absence of chattering). Also it is more robust to perform emergency maneuvers (for example, big turns). It is clear that with a greater consideration of the model and the characteristics of passivity of the system, the control by $I \& I$ can greatly improve the performances for autonomous driving applications and ADAS.

Stability of the system is assured with the proposed $I \& I$ controller for all its gains $\lambda>0$ and $K>0$. This is a very strong result that reveals some interesting characteristics of the system. More deep and promising characteristics of the system are under study now by the authors. Moreover, we will also test these control laws on a semi-autonomous vehicleour robotized vehicle, in the laboratory Heudiasyc.

\section{REFERENCES}

[1] G. Tagne, R. Talj, and A. Charara, "Higher-Order Sliding Mode Control for Lateral Dynamics of Autonomous Vehicles , with Experimental Validation," in Int. IEEE Conference on Intelligent Vehicles Symposium (IV), (Gold Coast), pp. 678-683, 2013.
[2] J. Levinson, J. Askeland, J. Becker, J. Dolson, D. Held, S. Kammel, J. Z. Kolter, D. Langer, O. Pink, V. Pratt, M. Sokolsky, G. Stanek, D. Stavens, A. Teichman, M. Werling, and S. Thrun, "Towards Fully Autonomous Driving : Systems and Algorithms," in Int. IEEE Conference on Intelligent Vehicles Symposium (IV), (Baden-Baden), pp. 163-168, 2011.

[3] A. Broggi, M. Bertozzi, and A. Fascioli, "The ARGO autonomous vehicle's vision and control systems," Int. Journal of Intelligent Control and Systems, vol. 3, no. 4, pp. 409-441, 1999.

[4] P. Zhao, J. Chen, T. Mei, and H. Liang, "Dynamic motion planning for autonomous vehicle in unknown environments," in Int. IEEE Conference on Intelligent Vehicles Symposium (IV), 2011.

[5] R. Marino, S. Scalzi, and M. Netto, "Nested PID steering control for lane keeping in autonomous vehicles," Control Engineering Practice, vol. 19, pp. 1459-1467, Dec. 2011.

[6] S. Hima, B. Lusseti, B. Vanholme, S. Glaser, and S. Mammar, "Trajectory Tracking for Highly Automated Passenger Vehicles," in International Federation of Automatic Control (IFAC) World Congress, (Milano), pp. 12958-12963, 2011.

[7] R. Rajamani, Vehicle dynamics and control. Springer, 2006.

[8] A. Benine-Neto, S. Scalzi, S. Mammar, and M. Netto, "Dynamic controller for lane keeping and obstacle avoidance assistance system," in Int. IEEE Conference on Intelligent Vehicles Symposium (IV), pp. $1363-1368,2010$.

[9] J. Naranjo, C. Gonzalez, R. Garcia, and T. de Pedro, "Lane-Change Fuzzy Control in Autonomous Vehicles for the Overtaking Maneuver," Iint. IEEE Transactions on Intelligent Transportation Systems, vol. 9, pp. 438-450, Sept. 2008.

[10] B. Soualmi, C. Sentouh, J. C. Popieul, and S. Debernard, "Fuzzy Takagi-Sugeno LQ controller for lateral control assistance of a vehicle," in Int. IEEE Conference on Intelligent Vehicles Symposium (IV), (Alcalá de Henares), pp. 377-382, 2012.

[11] D. Kim, J. Kang, and K. Yi, "Control strategy for high-speed autonomous driving in structured road," in Int. IEEE Conference on Intelligent Transportation Systems (ITSC), 2011.

[12] L. Nehaoua and L. Nouvelière, "Backstepping based approach for the combined longitudinal-lateral vehicle control," in Int. IEEE Conference on Intelligent Vehicles Symposium (IV), (Alcalá de Henares), pp. 395-400, 2012.

[13] T. Besselmann and M. Morari, "Autonomous Vehicle Steering Using Explicit LPV-MPC," in European Control Conference (ECC), (Budapest), pp. 2628-2633, 2009.

[14] P. Hingwe and M. Tomizuka, "Experimental evaluation of a chatter free sliding mode control for lateral control in AHS," in American Control Conference (ACC), 1997.

[15] S. Chaib, M. Netto, and S. Mammar, "H inf, adaptive, PID and fuzzy control: a comparison of controllers for vehicle lane keeping," in Int. IEEE Intelligent Vehicles Symposium (IV), pp. 139-144, 2004.

[16] D. Heß, M. Althoff, and T. Sattel, "Comparison of Trajectory Tracking Controllers for Emergency Situations," in Int. IEEE Conference on Intelligent Vehicles Symposium (IV), (Gold Coast), pp. 163-170, 2013.

[17] L. Menhour, D. Koenig, and B. Andr, "Continuous-Time and DiscreteTime Switched H inf. State Feedback Controllers : Application for a Robust Steering Vehicle Control," in European Control Conference (ECC), (Zurich), pp. 986-991, 2013.

[18] A. Astolfi, D. Karagiannis, and R. Ortega, Nonlinear and Adaptive Control with Applications. Springer, 2008.

[19] P. Kokotovic and R. Marino, "On vanishing stability regions in nonlinear systems with high-gain feedback," IEEE Transactions on Automatic Control, vol. 31, pp. 967-970, Oct. 1986. 